\title{
Medidas para competitividade: fundamentos, críticas e abordagens
}

\author{
alternativas
}

\author{
Bruno César Araújo*
}

\begin{abstract}
RESUMO - No mês de janeiro é divulgado oficialmente o indicador de competitividade mais famoso, o Índice de Competitividade Global (GCI), do Fórum Econômico Mundial (WEF). De modo similar, o Institute for Management Development (IMD) lança também o seu ranking, construído a partir do World Competitiveness Scoreboard (WCS). Nos últimos anos, as notícias não foram muito animadoras para o Brasil. Mas até que ponto devem nos preocupar comparações como o GCI ou o WCS? Exercícios de benchmarking internacional como os propostos por estes índices certamente são válidos, desde que seus resultados sejam tão discutidos quanto suas limitações. Delineamos cinco críticas a estes indicadores, duas de natureza prática e três de natureza teórica. Ao final do texto, propomos uma abordagem analítica e dinâmica para comparações internacionais, tendo por base as pesquisas de inovação, em alternativa aos exercícios sintéticos e estáticos que são o GCI e o WCS.
\end{abstract}

Palavras-chave: Competitividade. Indicadores de competitividade. Pesquisas de inovação. Estratégias competitivas.

\section{MEDIDAS PARA COMPETITIVIDADE: FUNDAMENTOS E ABORDAGENS ALTERNATIVAS}

No mês de janeiro ocorre o Fórum Econômico Mundial (World Economic Forum WEF) ocasião na qual é lançado oficialmente o Relatório de Competitividade Global (Global Competitiveness Report). Do relatório, a principal atração é o Índice de Competitividade global (GCI), do qual é possível elaborar um ranking de competitividade para diferentes nações ou regiões. De modo similar, o Institute for Management Development (IMD), sediado na cidade de Lousanne, também na Suíça, lança anualmente seu Relatório de Competitividade Mundial (Word Competitiveness Yearbook), que conta também com seu ranking, construído a partir do World Competitiveness Scoreboard (WCS). Com respeito a estes índices - os quais, aliás, foram efetivamente os mesmos até meados da década de 1990 - as notícias não têm sido tão animadoras para o Brasil nos últimos anos. No caso do GCI, a posição brasileira caiu de $45^{\circ}$

\footnotetext{
* Pesquisador do Instituto de Pesquisa Econômica Aplicada (IPEA). Endereço Eletrônico: bruno.araujo@ipea.gov.br. O autor agradece os comentários de João Alberto De Negri, Danilo Coelho, Lenita Maria Turchi, Gilson Geraldino, Divonsir Gusso e o auxílio de Patrick Alves e Geovane Lopes para a lide com os dados. Todos os erros e omissões são de responsabilidade exclusiva do autor.
} 
lugar em 2002 para 54 em 2003, $57^{\circ}$ em 2004, manteve esta posição em 2005, mas em 2006 nossa economia foi rebaixada para a $66^{a}$ posição e continuou a cair em 2007 , indo para a $72^{a}$ posição, ainda que seja preciso notar que durante o período foram inclusos mais países na comparação, e que o Brasil vem mantendo uma posição mediana (literalmente) desde 2002. Diante destes resultados, pergunta-se: até que ponto os policy makers devem se preocupar com comparações como o GCI ou o WCS?

Para responder a esta pergunta, primeiro é interessante recordar o conceito de competitividade sobre o qual a construção dos índices se baseia. O WEF define competitividade em seu relatório como o "conjunto de fatores, políticas e instituições que determinam o nível de produtividade de um país" (WEF, 2007), enquanto o IMD sustenta que "[os analistas da] competitividade avaliam como as nações e as empresas administram a totalidade de suas competências a fim de atingir prosperidade e lucro" (IMD, 2007). Em ambos os casos, o conceito se relaciona com uma idéia de vantagem absoluta das nações ligada ao nível global de produtividade, e se inspira fortemente nas idéias de Michael Porter em seu livro intitulado "A vantagem competitiva das nações" (Porter, 1989). Entretanto, há uma forte controvérsia acerca da aplicabilidade do conceito de competitividade para as nações, e esta é a primeira fonte das críticas a comparações como o GCI.

Mesmo que concordemos com a idéia de comparar nações a partir do nível global de produtividade, instituições, clima de investimento e outras, o ponto é que indicadores como o GCI ou WCS apresentam limitações que mereceriam ser tão debatidas quanto seus resultados. No presente texto, delineamos cinco críticas - duas de natureza prática e três de natureza teórica - à maneira como estes indicadores são construídos.

\section{OS ÍNDICES DE COMPETITIVIDADE MAIS FAMOSOS: O GCI E O WCS}

Como mencionado, tanto o GCI quanto o WCS seguem a mesma metodologia:

1 - Ambos são índices ex-ante de competitividade, uma vez que eles buscam não medir o desempenho dos países, mas fazer uma avaliação do que os formuladores dos índices pensam como "pilares" da competitividade;

2 - Sub-índices para estes "pilares" são construídos e posteriormente condensados em um escalar, o qual embasará o ranking de competitividade entre as nações;

3 - Ambos os índices combinam "dados duros” (hard data) como balança comercial, crescimento econômico, desemprego etc. com avaliações subjetivas oriundas de pesquisas de opinião junto a empresários, a fim de obter uma classificação para fatores da competitividade 
como qualidade das instituições, favoritismo em licitações, sofisticação dos negócios no país e outros.

Metodologia semelhante é aplicada em pesquisas como a do Banco Mundial, sobre clima de investimento. Estes índices são usados pela comunidade de negócios, a fim de avaliar clima de investimento, governos, políticas e o desempenho de outras nações, buscando benchmarks para suas políticas de desenvolvimento. São utilizados também por acadêmicos estudiosos da competitividade. A seguir, os rankings mais recentes de ambos os índices. De forma sintética, mostraremos os 10 primeiros países, a posição brasileira e a de alguns outros países selecionados.

TABELA 1 - RANKING DE COMPETITIVIDADE - GCI E WCS, PARA OS 10 PRIMEIROS, BRASIL E PAÍSES SELECIONADOS

\begin{tabular}{|c|c|c|c|c|}
\hline \multicolumn{3}{|c|}{ Growth Competitiveness Index } & \multicolumn{2}{|c|}{$\begin{array}{c}\text { World Competitiveness } \\
\text { Scoreboard }\end{array}$} \\
\hline $\begin{array}{c}\text { Ranking } 2008 \text { - } \\
2007\end{array}$ & $\begin{array}{c}\text { Ranking } 2008-2007 \\
\text { (dentre os países do } \\
\text { ranking anterior) }\end{array}$ & $\begin{array}{l}\text { Ranking 2007- } \\
2006\end{array}$ & Ranking 2007 & Ranking 2006 \\
\hline $1-\mathrm{EUA}$ & 1 & 1 & $1-\mathrm{EUA}$ & 1 \\
\hline 2 - Suíça & 2 & 4 & 2 - Cingapura & 3 \\
\hline 3 - Dinamarca & 3 & 3 & 3 - Hong Kong & 2 \\
\hline 4 - Suécia & 4 & 9 & 4 - Luxemburgo & 9 \\
\hline 5 - Alemanha & 5 & 7 & 5 - Dinamarca & 5 \\
\hline 6 - Finlândia & 6 & 6 & $6-$ Suíça & 8 \\
\hline 7 - Cingapura & 7 & 8 & 7 - Islândia & 4 \\
\hline 8 - Japão & 8 & 5 & 8 - Holanda & 15 \\
\hline 9 - Reino Unido & 9 & 2 & 9 - Suécia & 14 \\
\hline 10 - Holanda & 10 & 11 & 10 - Canadá & 7 \\
\hline 26 - Chile & 26 & 27 & 26 - Chile & 23 \\
\hline 40 - Portugal & 38 & 43 & 38 - Colombia & 34 \\
\hline 52 - México & 49 & 52 & 39 - Portugal & 37 \\
\hline 69 - Colombia & 65 & 63 & 47 - México & 45 \\
\hline 72 - Brasil & 68 & 66 & 49 - Brasil & 44 \\
\hline 85 - Argentina & 79 & 79 & 51 - Argentina & 47 \\
\hline 131 - Chade & 122 & 121 & 55 - Venezuela & 53 \\
\hline Países analisados & Países analisados & Países analisados & Países analisados & Países analisados \\
\hline 131 & 122 & 122 & 55 & $55+$ regiões \\
\hline
\end{tabular}

FONTE: Elaboração própria a partir de WEF (2007) e IMD (2007).

A despeito das semelhanças com respeito à abordagem - o que na prática resulta em uma correlação de ranking de Spearman de 0,88 - a construção dos índices apresenta diferenças sutis. A primeira diz respeito ao número de pilares da competitividade considerados. O GCI considera 12 pilares (instituições, infra-estrutura, macroeconomia, saúde e educação básica, educação superior e treinamento, eficiência no mercado de bens, eficiência 
no mercado de trabalho, sofisticação do mercado financeiro, prontidão tecnológica, tamanho do mercado, sofisticação dos negócios e inovação), enquanto o WCS considera apenas 4 (performance econômica, eficiência governamental, eficiência empresarial e infra-estrutura).

Outra característica distintiva do GCI é que, além de se basear em mais indicadores, ele dá ponderações diferentes aos pilares de acordo com o nível de renda per capita. Por exemplo, a ponderação dos pilares da competitividade nos países que estão no quartil inferior de renda per capita dá mais peso aos quatro primeiros pilares. Além disso, para todos os países o GCI dá mais peso a variáveis relativas à inovação e capacidades inovativas. Por outro lado, o WCS apresentou até sua edição de 2006 indicadores de competitividade não apenas para países, mas também para regiões dentro dos países, como a Lombardia na Itália ou São Paulo no Brasil (ainda que as posições relativas destas regiões com respeito a seus países não fossem muito diferentes - por exemplo, São Paulo ficou na $48^{a}$ posição naquele ano e o Brasil como um todo na $\left.52^{\mathrm{a}}\right)$.

O grande apelo popular que gozam indicadores como o GCI ou o WCS se deve ao fato de que eles provêm uma medida ampla, porém relativamente simples, de indicadores de competitividade ex-ante dos países, de forma comparável entre eles, e que pode ser útil, pelo menos em um primeiro momento, para análises de benchmarking. Contudo, há algumas fraquezas com respeito à forma pela qual os índices são construídos. Exporemos nas subseções a seguir cinco fraquezas do índice, duas de natureza prática e três de natureza teórica.

\subsection{FRAQUEZAS PRÁTICAS}

2.1.1 Baixa correlação entre os índices e crescimento observado

Surpreendentemente, o GCI - que em 2006 havia atribuído ao "G" de sua sigla para a palavra crescimento (growth) - não parece guardar correlação com o crescimento, nem mesmo no médio prazo. Testamos a correlação de Pearson entre o crescimento real per capita entre 2000 e 2004 e a posição no ranking do país em 2000, e esta se revelou extremamente baixa: apenas 0,083. Ainda que não tenhamos conduzido análise semelhante para o WCS, dada sua alta correlação com o GCI os resultados devem ser bastante semelhantes. A seguir, mostramos o diagrama de dispersão do exercício, bem como uma correlação da posição no ranking, não com o crescimento, mas com o nível de renda per capita em 2000. 
GRÁFICO 1 - DIAGRAMAS DE DISPERSÃO: POSIÇÃO NO RANKING EM 2000 VS. CRESCIMENTO DE RENDA PER CAPTA REAL ENTRE 2000-2004

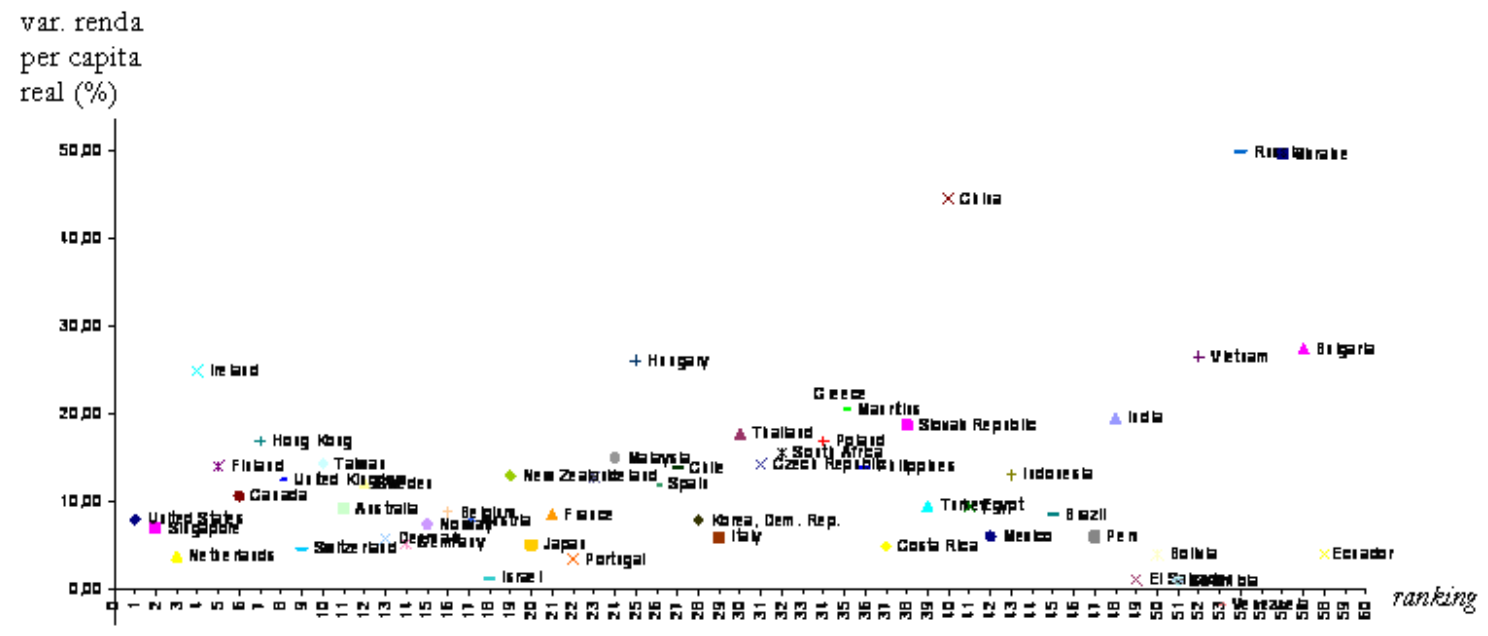

FONTE: Os dados para renda per capita real dos países foram obtidos de Heston, Summers e Aten (2006), enquanto os dados do ranking do ano de 2000 foram obtidos do relatório World Competitiveness Report do ano de 2001. A renda per capita real é medida em dólares PPC de 2000. GRÁFICO 2 - DIAGRAMA DE DISPERSÃO: POSIÇÃO NO RANKING EM 2000 VS. RENDA PER
CAPTA REAL EM 2000

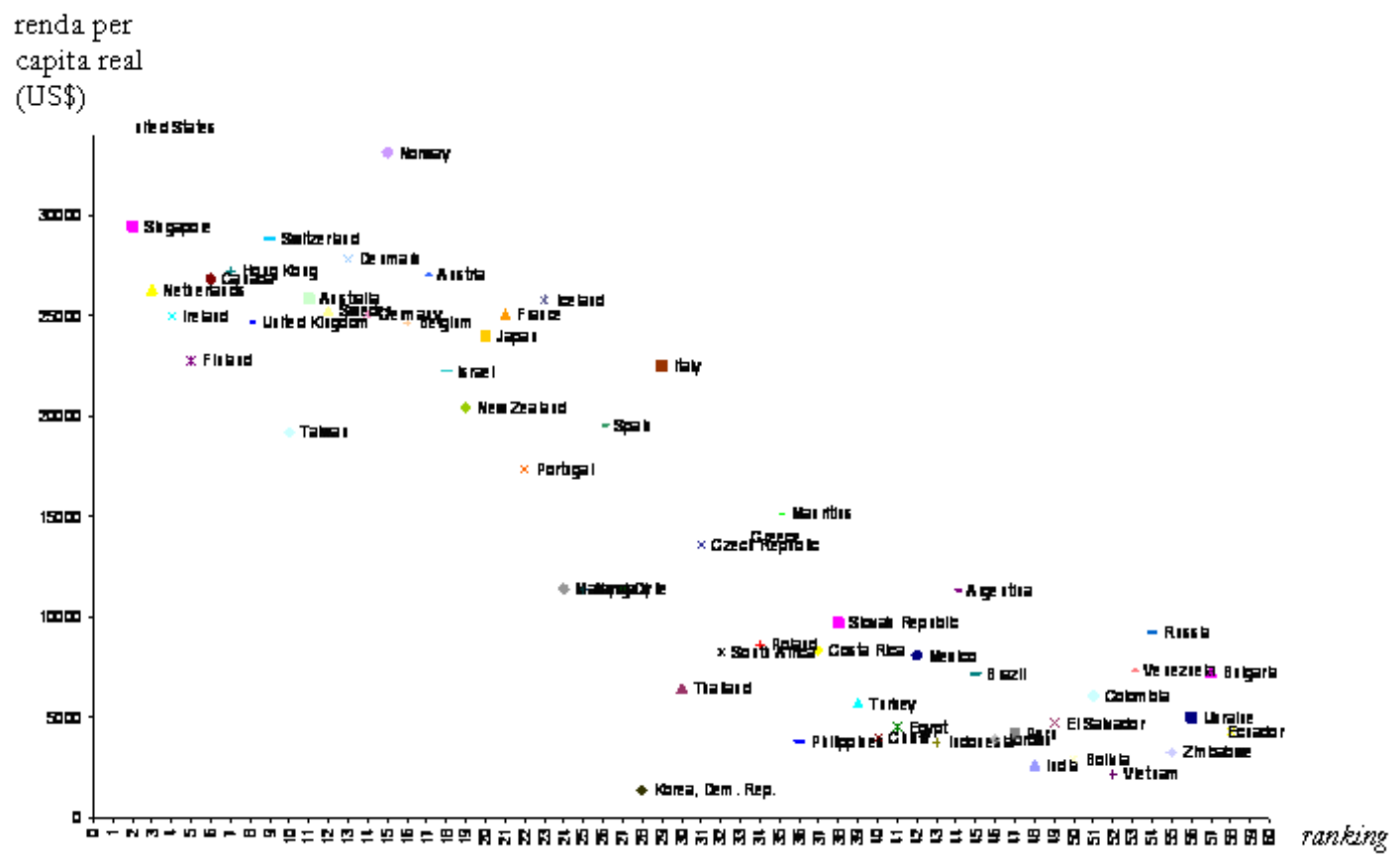

FONTE: Os dados para renda per capita real foram obtidos de Heston, Summers e Aten (2006), enquanto os dados do ranking do ano de 2000 foram obtidos do relatório World Competitiveness Report do ano de 2001. A renda per capita real é medida em dólares PPC de 2000.

Talvez esta ausência de correlação com o crescimento se deva ao fato do índice, por se basear fortemente em dados que provêem de uma pesquisa de opinião junto a homens de 
negócios, represente mais uma medida do risco de se fazer negócios no país do que de potencial de crescimento em si - daí sua alta correlação com o nível inicial de renda per capita $(-0,882$, para o ano de 2000).

2.1.2 A conjuntura afeta muito os índices e, os países mudam rapidamente suas pocições de um ano para o outro

A despeito da correlação de ranking de Spearman ser bastante alta de um ano para o outro (entre 2006 e 2007, ela é de 0,98) ou mesmo entre prazos mais longos (entre 2000 e 2007, ela é de 0 ,90), não raro países se movem 10 posições no ranking de um ano para o outro, o que talvez não seja um resultado plausível, ao se considerar os determinantes estruturais da competitividade.

$\mathrm{Na}$ verdade, as mudanças de um ano para o outro parecem estar associadas ao fato de que a conjuntura afeta sensivelmente a posição dos países. Por exemplo, os países que sistematicamente apresentam as melhores posições no pilar macroeconômico não são exatamente países conhecidos pela qualidade institucional de suas políticas macroeconômicas, e sim, por serem grandes países exportadores de petróleo - Kwait, Argélia, Arábia Saudita e Líbia, os quais vêm obtendo superávits fiscais crescentes desde a contínua subida dos preços do petróleo (e dos royalties pagos ao governo) iniciada em 2003.

A despeito de todos os esforços que o Brasil vem fazendo desde 1999 para ajustar as contas públicas e reduzir a relação dívida/PIB, nosso país obteve a $126^{a}$ posição no ranking de 2007 no tocante ao pilar macroeconômico, bem atrás de México (35), Bolívia (49), Venezuela (70) ou mesmo Bangladesh (87).

\subsection{FRAQUEZAS TEÓRICAS}

\subsubsection{Não há intertemporalidade na análise}

Alguns indicadores macroeconômicos baseados em hard data são tomados ano a ano quando, do ponto de vista teórico, deveriam ser analisados em uma perspectiva intertemporal. Isto evitaria, inclusive, que a conjuntura afetasse substancialmente a classificação no ranking.

Vejamos o caso do balanço fiscal e do balanço comercial. Quanto ao último, como já comentado, um alto superávit comercial em um dado ano pode ser um sintoma de recessão econômica, ou mesmo o resultado de políticas protecionistas e anti-competitivas. Por sua vez, uma política macroeconômica contra-cíclica pode ser pró-competitiva, ainda que possa 
conduzir a um déficit comercail. Estes indicadores, no mínimo, deveriam ser analisados de acordo com um horizonte mais longo.

\subsubsection{Os critérios de ponderação entre hard data e soft data não são claros}

Como já mencionado, ambos os índices misturam hard data, de instituições que provêem estatísticas econômicas, com soft data de uma pesquisa conduzida junto a homens de negócios, acerca de itens não mensuráveis por meio de hard data, tão diversos quanto independência do judiciário e comportamento ético das firmas. No caso do GCI o problema é que, do total de 113 indicadores analisados, apenas 32 são hard data, e têm o mesmo peso dos indicadores do tipo soft data.

O problema sobre como ponderar hard data com soft data poderia ser facilmente resolvido se houvesse uma medida de competitividade amplamente reconhecida. Neste caso, por meio de técnicas multivariadas um pesquisador poderia achar os pesos ótimos para os diversos indicadores do GCI, buscando se aproximar desta medida de competitividade.

Porém, se por um lado não há esta medida amplamente reconhecida, por outro lado é pouco razoável acreditar que atribuir o mesmo peso para os indicadores seja a solução ótima. A título de exemplo, no $9^{\circ}$ pilar do GCI, qual seja, a prontidão tecnológica, os "dados duros", tais como número de aparelhos celulares, de usuários de internet, de PCs e de assinantes de serviços de banda larga são postos em pé de igualdade com soft data como disponibilidade tecnológica, absorção tecnológica por parte das firmas, marco regulador de tecnologias de informação e comunicação e investimento estrangeiro direto e transferência de tecnologia. O WCS atribui mais peso aos hard data, mas também padece do mesmo problema, qual seja, a não garantia de que esta seja uma solução ótima (ainda que mais razoável).

\subsubsection{Pré-condições não necessariamente se materializam em desempenho}

Por fim, esta é a última crítica às tentativas de se mensurar competitividade ex-ante: não necessariamente as pré-condições para a competitividade se convertem na mesma. Aliás, o próprio Porter em seu livro sustenta, com diversos exemplos, que não raro desvantagens iniciais em certa indústria podem ser um motivador para a inovação e competitividade.

\section{RUMO A ABORDAGENS ALTERNATIVAS}

Discutidas as críticas aos índices de competitividade mais famosos, resta a pergunta da introdução do texto: até que ponto os policy makers devem se preocupar com comparações como o GCI ou o WCS? De forma alguma, sugerimos neste texto que exercícios de 
benchmarking internacional como estes devem ser abandonados por completo. Apenas sugerimos que estes exercícios devem ser compreendidos de acordo com suas limitações. E, sobretudo, complementado por comparações baseadas em medidas de desempenho e capacidades inovativas dos tecidos produtivos dos países.

Esta é a linha adotada nos projetos de pesquisa do IPEA na área de comparações internacionais, consubstanciado até agora nos livros Technological Innovation in Brazilian and Argentine Firms ${ }^{36}$ e Innovation in Braril, Mexico and Argentina: what can we learn from microdata?. ${ }^{37}$ Em ambos os livros, foi empregada uma classificação de empresas de acordo com suas estratégias competitivas com respeito à inovação tecnológica.

A classificação criada permite-nos distinguir e analisar três estratégias competitivas básicas por parte das empresas latino-americanas como reação à abertura econômica nos anos 90, a partir de indicadores-chave:

1. Firmas que reagiram proativamente, investindo em inovação tecnológica e diferenciação de produtos como estratégia competitiva;

2. Firmas que fizeram um ajuste apenas parcial nos processos produtivos a fim de competir com os produtos importados, principalmente via aquisição de maquinário e inovações de processo;

3. Firmas que são menos inovativas e menos produtivas, que sobrevivem operando em escala em geral ineficiente e explorando mercados locais, não raro explorando vantagens de baixo custo de mão-de-obra.

Lembremos que o cerne das vantagens competitivas reside nas estratégias competitivas baseadas em inovação e diferenciação de produtos. Contudo, dentro de um mesmo país podem coexistir firmas que adotam diferentes estratégias competitivas, o que pode tornar difícil a síntese da competitividade de um país em um simples escore. E é exatamente neste ponto que reside a maior riqueza desta abordagem: ela é analítica e estrutural, pois analisa a estrutura industrial como um todo; em contraposição às análises do tipo GCI e WCS, que são sintéticas e estáticas, pois buscam um simples escore que resuma toda a estrutura produtiva do país, mas são fortemente influenciadas pela conjuntura, como vimos.

\footnotetext{
${ }^{36}$ De Negri e Turchi (2007).

${ }^{37}$ De Negri e Araújo (2008).
} 
No Brasil, em estudo prévio de Salerno e De Negri (2005) buscaram-se três indicadores-chave para distinguir as estratégias competitivas das firmas, a saber: a atividade exportação, a auferição de preço-prêmio para suas exportações, e o nível de produtividade das firmas. Naquele estudo, as firmas industriais brasileiras foram classificadas da seguinte forma:

A) Firmas que inovam e diferenciam produtos - empresas que realizaram inovação de produto para o mercado e obtiveram preço-prêmio acima de 30\% nas suas exportações quando comparadas com as demais exportadoras brasileiras do mesmo produto. Nesse grupo estão incluídas, portanto, as firmas que adotam estratégias competitivas mais vantajosas, e compõem o segmento mais dinâmico que, apesar de constituir a minoria no número de firmas, tende a capturar parcela maior da renda gerada pela indústria.

B) Firmas especializadas em produtos padronizados, cuja estratégia competitiva impõe que o foco de sua atuação seja a redução de custos, ao invés da criação de valor como na categoria acima - são consideradas aqui as firmas exportadoras não incluídas na categoria acima e as não exportadoras que apresentam eficiência igual ou maior do que as firmas que exportam nesta categoria. Tendem a ser atualizadas do ponto de vista de características operacionais como fabricação, gestão da produção, gestão da qualidade de conformação e logística, que são imperativos para sustentação de custos relativamente mais baixos, mas na média estão defasadas, relativamente à categoria anterior, no que se refere a outras armas da competição como pesquisa e desenvolvimento, marketing e gerenciamento de marcas.

C) Firmas que não diferenciam produtos e têm produtividade menor, ou seja, demais firmas que não pertencem às categorias anteriores - abarca as empresas tipicamente não exportadoras, menores, que podem inclusive inovar, mas são menos eficientes nos mais variados sentidos, que se mostram capazes de captar espaços em mercados menos dinâmicos através de baixos preços e outras possíveis vantagens.

Para as comparações internacionais, não foi possível contar com a variável preçoprêmio da firma. O preço prêmio é uma variável importante para comprovação da hipótese de diferenciação de produto da firma. Os gastos em P\&D como proporção do faturamento da empresa foram uma alternativa à ausência desta variável uma vez que estes gastos são fortemente correlacionados com a capacidade de a empresa diferenciar produtos. Levando em conta esta ponderação, os indicadores-chave usados para a classificação resultaram nas seguintes categorias:

$\mathrm{X}$ - Firmas que inovaram produto novo para o mercado, exportaram e gastaram mais em P\&D do que a média do seu setor industrial (média dos três países); 
Y -Demais exportadoras não incluídas no grupo X e firmas não exportadoras que têm produtividade do trabalho superior à média do seu setor industrial (média dos três países);

Z - Demais firmas não incluídas nos grupos acima.

Esta classificação é análoga à anterior, ou seja, no primeiro grupo $(\mathrm{X})$ estariam as empresas que inovam e diferenciam seus produtos, no segundo grupo (Y), estariam as empresas especializadas em produtos padronizados, e no terceiro grupo (Z) estariam as empresas que têm problemas de produtividade, eficiência e que não diferenciam produto. Algumas tabulações obtidas na comparação estão a seguir na tabela 2, a título de exemplo da metodologia desenvolvida.

Dois pontos interessantes podem ser destacados da tabela acima. Além de a produção industrial brasileira ser 4 vezes a argentina e aproximadamente 1.6 vezes a mexicana, nota-se que a escala de produção das empresas brasileiras é muito maior, e isto especialmente verdadeiro quando se trata da primeira categoria, a que inova e diferencia produtos. De fato, o Brasil é o país onde as empresas que inovam e diferenciam produto têm a maior parcela das vendas industriais $(25,19 \%)$ e das exportações $(33,16 \%)$. Outro ponto que merece comentário é a distribuição de produtividade entre as categorias. Tanto no Brasil quanto na Argentina a categoria mais produtiva é composta pelas firmas inovadoras e que diferenciam produtos, mas o mesmo não ocorre no México. Neste país, a especialização em produtos estandardizados parece a estratégia competitiva mais promissora. As firmas argentinas têm o nível de produtividade mais alto entre os três países, mas é preciso lembrar que a Argentina adotava um regime de paridade fixa até 2001, e entre 1998-2001 o país viveu o ápice de sua crise econômica, situação em que as firmas menos produtivas simplesmente deixaram de existir. 
TABELA 2 - NÚMERO DE FIRMAS, ESCALA PRODUTIVA E PRODUTIVIDADE D4E FIRMAS INDUSTRIAS BRASILEIRAS (2000), ARGENTINAS (2001) E MEXICANAS (2000)

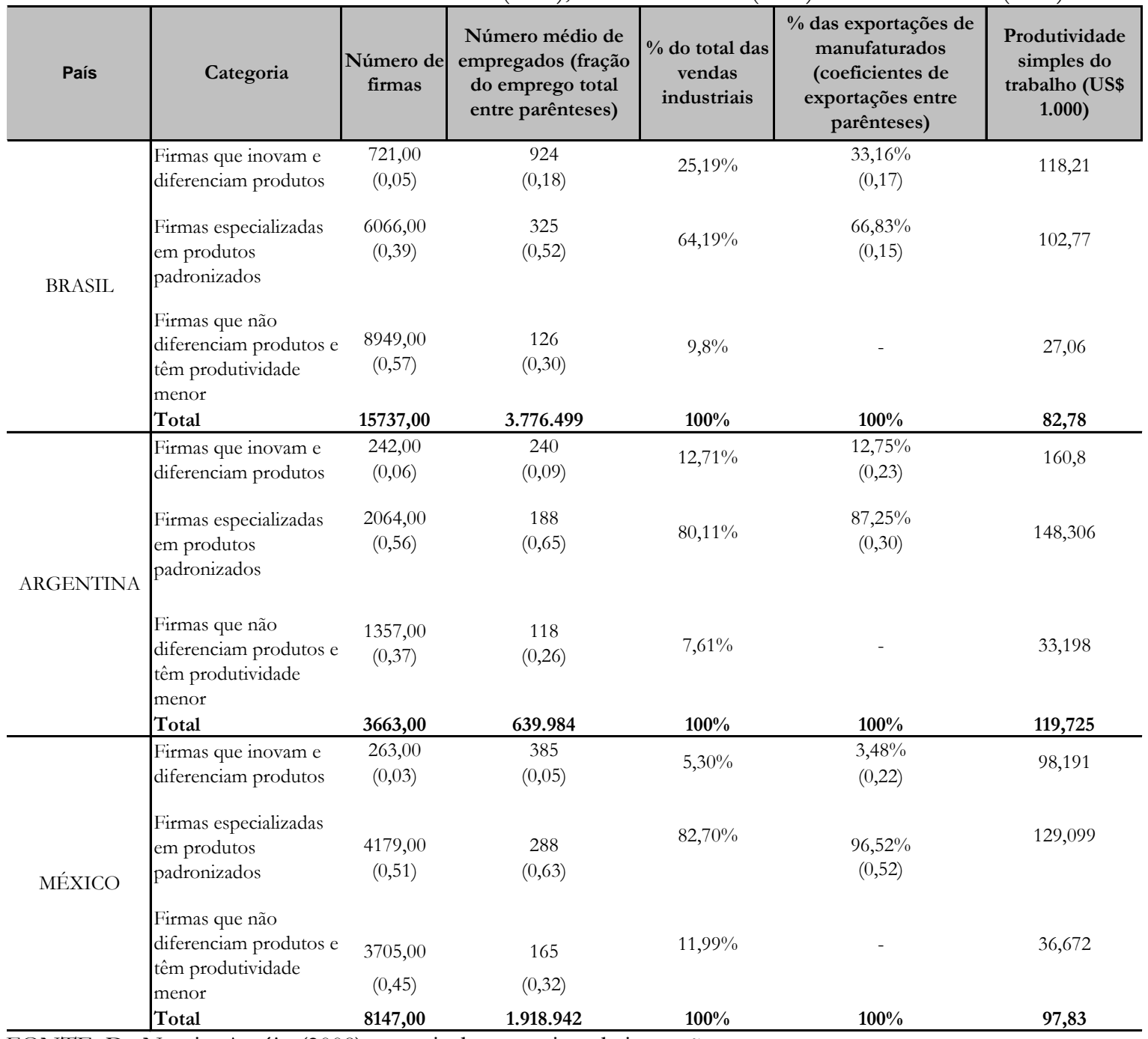

FONTE: De Negri e Araújo (2008), a partir das pesquisas de inovação.

Não é objetivo do presente texto a discussão profunda acerca dos resultados obtidos nesta comparação, ficando a cargo do leitor interessado consultar as referências bibliográficas. Contudo, é fácil notar que o potencial dos exercícios de benchmarking internacional é muito superior quando combinam tanto indicadores ex-ante de competitividade quanto indicadores e análises mais estruturais, que buscam captar desempenho, limitações e oportunidades do tecido industrial, especialmente no que tange a inovação - verdadeiramente, o motor da competitividade das nações. 


\section{REFERÊNCIAS}

De NEGRI, J. A. e SALERNO, M. S. Inovações, Padrões Tecnológicos e Desempenho das Firmas Industriais Brasileiras. Brasília: IPEA, 2005.

De NEGRI, J. A. e TURCHI, L. M. Technological Innovation in Brazilian and Argentine Firms. Brasilia: IPEA, 2007.

De NEGRI, J. A. e ARAÚJO, B. C. Innovation in Brazil, Mexico and Argentina: what can we learn from microdata? Brasilia: IPEA, 2008 (no prelo).

DUNNING, J. “Think again Professor Krugman: competitiveness does matter." International Executive Vol 37, N. 4, 1995.

HESTON, A., SUMMERS, R. e ATEN, B. "Penn World Table Version 6.2." Center for International Comparisons of Production, Income and Prices at the University of Pennsylvania, 2006.

Institute for Management Development. World Competitiveness Yearbook. Lousanne: Institute for Management Development, 2007.

KRUGMAN, P. “Competitiveness: a dangerous obsession.” Foreign Affairs, Vol. 73, N. 2, 1994. . “A reply to Prof. Dunning”. International Executive Vol 37, N. 4, 1995.

PORTER, M. E. A Vantagem Competitiva das nações, Rio Janeiro: Campus, 1989. Vantagem competitiva: criando e sustentando um desempenho superior. Rio de Janeiro: Campus, 1985.

WORLD ECONOMIC FÓRUM. World Competitiveness Report. Davos: World Economic Forum, 2007 